ARTÍCULO ORIGINAL

Recibido:

03/07/2019

Aprobado:

31/10/2019

\title{
Análisis químico y de peligrosidad de lodos del sistema de tratamiento de aguas residuales en la industria de palma
}

\section{Chemical and hazard analysis of sludge of the wastewater treatment system in the industry of palma}

\begin{abstract}
Bastidas Chávez Pablo Ignacio
(1) https://orcid.org/0000-0003-4515-9309

Universidad Central del Ecuador

pibastidas@uce.edu.ec
\end{abstract}

\author{
Malacatus Cobos Paúl Nicandro \\ (1) https://orcid.org/0000-0003-2417-7348 \\ Universidad Central del Ecuador \\ pnmalacatus@uce.edu.ec
}

\author{
Chuquitarco Moreno Pablo Andrés \\ (1) https://orcid.org/0000-0001-6253-7450 \\ Universidad Central del Ecuador \\ pachuquitarco@uce.edu.ec
}

\section{RESUMEN}

El principal problema en los sistemas de tratamiento de aguas residuales de la industria de palma es la acumulación de lodos en las lagunas de oxidación, para extraer los lodos se instaló el geotubo, sin embargo, es necesario dar un destino final a los mismos. El objetivo de este trabajo fue analizar los componentes químicos y biológicos de los lodos generados en la laguna de oxidación $\mathrm{N}^{\circ} 4$ y en el geotubo del sistema de tratamiento de aguas, determinando la peligrosidad e identificando el destino final del mismo. Además, se estableció si el floculante utilizado en el paso del lodo de la laguna hacia el geotubo incide en sus características químicas. Para esto, se realizaron tres muestreos en tres diferentes días y meses. Para la laguna de oxidación $\mathrm{N}^{\circ} 4$ se recolectaron tres submuestras en diferentes puntos para formar una muestra compuesta y en el geotubo se tomaron muestras a 0,5 y $2 \mathrm{~m}$ de profundidad. Analizando parámetros agrícolas como NPK, se obtuvieron niveles altos de concentración de nitrógeno y fósforo, comparados con guías de fertilización, y niveles adecuados para el potasio, lo que indica que se puede emplear para uso agrícola. La corrosividad en base al pH del lodo está dentro del rango "no corrosivo" según la normativa. La reactividad, en base al nivel de cianuros se estableció como despreciable. La toxicidad por metales pesados, en cuanto a níquel, cromo, plomo y cadmio, resultaron bajo los niveles permisibles. La inflamabilidad de lodos, cualitativamente, se determinó que no registra presencia de gases inflamables. En el parámetro biológico infeccioso por concentración de coliformes totales, el resultado está bajo los límites permisibles. Finalmente, el lodo tanto de laguna como del geotubo resulta ser "no peligroso" según las normativas nacionales e internacionales y sus características químicas no son alteradas por el uso del floculante indicado.

\section{Palabras clave:}

lodos; laguna; geotubo; NPK; floculante.

\section{ABSTRACT}

The main problem in the wastewater treatment systems of the palm industry is the accumulation of sludge in the oxidation lagoons, to extract the sludge the geotube was installed, however, it is necessary to give a destination to them. The objective of this work was to analyze the chemical and biological components of the sludge generated in the oxidation lagoon No. 4 and in the geotube of the water treatment system, determining the danger and identifying its destination. In addition, it was established if the flocculant used in the passage of mud from the lagoon to the geotube affects its chemical characteristics. For this, three samples were taken in three different days and months. For the oxidation lagoon No. 4, three subsamples were collected at different points to form a composite sample, and samples at 0.5 and $2 \mathrm{~m}$ deep were taken in the geotube. Analyzing agricultural parameters such as NPK, high levels of nitrogen and phosphorus concentration were obtained, compared with fertilization guidelines, and adequate levels for potassium, indicating that it can be used for agricultural use. The corrosivity based on the $\mathrm{pH}$ of the sludge is within the "non corrosive" range according to the regulations. The reactivity based on the level of cyanides was established as negligible. Heavy metal toxicity, in terms of nickel, chromium, lead and cadmium, was below the permissible levels. The flammability of sludge, qualitatively, was determined not to register the presence of flammable gases. In the infectious biological parameter by concentration of total coliforms, the result is below the permissible limits. Finally, the mud of both the lagoon and the geotube turns out to be "non-hazardous" according to national and international regulations and its 
chemical characteristics are not altered using the indicated flocculant.

\section{Keywords:}

Sludge; lagoon; geotube, NPK; flocculant.

\section{INTRODUCCIÓN}

El procedimiento industrial que tiene por objeto la extracción de aceite crudo del fruto de palma aceitera conlleva a la generación de aguas residuales industriales, las mismas son tratadas en sistemas de tratamiento en base a lagunas de oxidación. Uno de los residuos que se generan en el tratamiento de aguas son los lodos.

En el atlas global para el manejo de excreta, lodos residuales y biosólidos, publicado en el 2008 por United Nations Human Settlements Programme y la Comisión Canadiense de manejo de lodos, especifica que los países en vías de desarrollo no consideran los lodos residuales como un residuo a tratar por lo que se tiene una nula inversión en su manejo, tratamiento y disposición final (Pérez, 2016). Generalmente, se toman medidas temporales de manejo que a futuro provocan problemas adicionales como disminución de la eficiencia del tratamiento de efluentes y sanciones por parte de organismos de control ambiental. Con esta investigación se quiere atender a estos problemas en la empresa de extracción de aceite de palma, enfocándose en los lodos de la laguna de oxidación $\mathrm{N}^{\circ} 4$ y el geotubo que almacena el lodo como un apoyo al tratamiento, teniendo en cuenta que en la mayoría de las industrias estos lodos se los elimina descargándolos a cursos de agua superficiales, sin considerar sus características físicas, químicas y biológicas que a menudo no están acorde a las normas relativas al cuidado del ambiente (Chisaguano, Malacatus y Paredes, 2017).

Los lodos generados en las plantas de tratamiento de efluentes industriales representan el 0,3\% al 1\% del volumen del agua tratada y en algunos casos llega hasta el $10 \%$ del volumen de agua tratada (Instituto Mexicano de tecnología del agua, 2000). En la investigación se determinó la humedad del lodo para cálculos y correcciones en muestra seca de los datos analizados.

Es necesario resaltar que la gestión y tratamiento de este tipo de residuos no solo conlleva un beneficio ambiental, sino también a un beneficio administrativo yeconómico. Si estos lodos pueden ser aprovechados como fertilizante según sus componentes sin que el floculante utilizado afecte su uso en el área agrícola. por todo esto la valorización de este residuo es importante, tanto en la recirculación y optimización del proceso como en el aprovechamiento en otras aplicaciones prácticas.

La extractora de aceite crudo de palma ha venido desarrollando actividades de remediación para el medio ambiente, buscando día a día metodologías a fin de evitar la contaminación con los desechos que produce. La planta de tratamiento de los efluentes consta de 4 lagunas de oxidación, por las cuales, pasan las aguas y sedimentos que salen del proceso de extracción de aceite de palma, el final del tratamiento es la laguna $\mathrm{N}^{\circ} 4$ que retiene bastantes sedimentos, se puede apreciar islas de lodos cuando baja el nivel de agua de un color pardo oscuro, indicio de material orgánico, en la superficie se aprecia la capa de una especie de musgo, por ello el dispositivo para disminuir la colmatación de los lodos es el geotubo instalado, por bombeo el lodo es traspasado al geotubo, en ese traslado se inyecta una concentración del polímero (poliacrilamida de alto peso molecular) para la floculación de sólidos suspendidos (Cogollo, 2010). El geotubo retiene y deshidrata el lodo para luego establecer el destino final del lodo. El problema en la planta de tratamiento es la colmatación de lodos que se han sedimentado y sobre todo en la laguna $N^{\circ} 4$, para solventar este problema se instaló el geotubo como apoyo al sistema de tratamiento. En la presente investigación se realizó los análisis del lodo de la laguna $\mathrm{N}^{\circ} 4$ y del lodo retenido en el geotubo, con la toma de muestras y midiendo parámetros nutricionales con un análisis de NPK, y los parámetros de corrosividad en base al $\mathrm{pH}$, reactividad, toxicidad por metales pesados, inflamabilidad y biológico infeccioso por número de coliformes totales, de este modo determinando si el lodo es peligroso y si el polímero utilizado causa alguna afectación al medio ambiente. Además, establecer recomendaciones y alternativas de aprovechamiento en la disposición final de los lodos, beneficio tanto para la empresa como para la población en general.

\section{MATERIAL Y MÉTODOS}

La extractora de aceite de palma se encuentra ubicada en la provincia de Esmeraldas, Cantón de Quinindé (Figura 1). Los lodos residuales se recolectaron en la laguna de oxidación $\mathrm{N}^{\circ} 4$ y en el geotubo del sistema de tratamiento de aguas durante 3 diferentes días y meses (Figura 2).

El objetivo del muestreo es recolectar una muestra representativa de los lodos en las dos zonas críticas que son: la laguna y el geotubo, que sea lo suficientemente pequeña para ser transportada y lo suficientemente grande para propósitos analíticos. (UNASAM - FCAM, 2009).

Se recolectó la muestra en la laguna de oxidación $\mathrm{N}^{\circ} 4$ de la siguiente manera: cada día de muestreo se tomó una submuestra en tres diferentes puntos cerca de las orillas de la laguna en sitios al azar con forme lo establecido en la (Secretaría de Ambiente del Municipio de Quito, 2014), cada una de un kilogramo aproximadamente, para ser analizado en el laboratorio. Una vez recolectado las tres submuestras, se crea una muestra compuesta de tal manera que pueda ser representativa (Muestra 3). Este procedimiento se repitió en los tres muestreos realizados a semana seguida,seguida. Es decir, se recolectó en la laguna de oxidación $\mathrm{N}^{\circ} 4$ un total de 9 submuestras de las cuales tres muestras compuestas fueron para los análisis de laboratorio (Figura 3).

En el geotubo, por su tamaño, altura y por la 


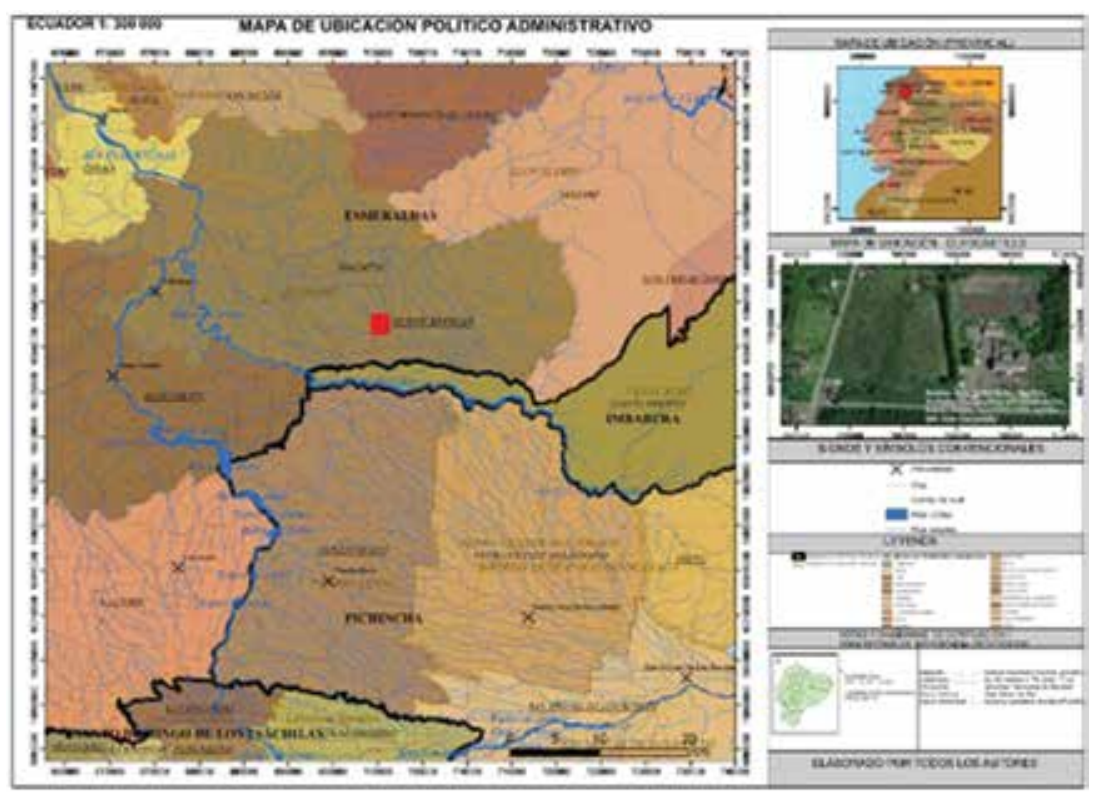

Figura 1. Mapa de ubicación de la extractora de aceite de palma. Prov. Esmeraldas



Figura 2. Sistema de tratamiento de aguas residuales

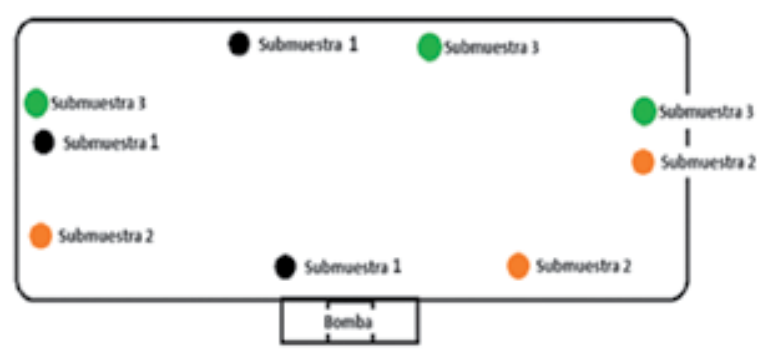

Figura 3. Esquema de muestreo en la laguna de oxidación $N^{\circ} 4$

abertura de recolección de las muestras, cada día de muestreo se tomaron muestras en dos puntos; uno en la parte superior a 0,5 m de profundidad (Muestra 1) y en la parte inferior del mismo a unos 2 metros de profundidad (Muestra 2), cada una de un kilogramo aproximadamente. Es decir, se recolectó en el geotubo un total de 6 muestras simples las mismas que fueron al laboratorio para análisis (Figura 4).



Figura 4. Esquema de muestreo en el geotubo

Todas las muestras fueron almacenadas en fundas ziploc, limpias, con cierre hermético para evitar cualquier riesgo de derrames y mezcla de las mismas. Cada muestra se etiquetó de la siguiente manera:

\section{Laguna de oxidación $\mathrm{N}^{\circ} \mathbf{4}$}

1. Muestra 1: $0,5 \mathrm{~m}$ de profundidad en el geotubo

2. Muestra 2: $2 \mathrm{~m}$ de profundidad en el geotubo

3. Muestra 3: Formación de la muestra compuesta por las tres submuestras tomadas en la laguna de oxidación $\mathrm{N}^{\circ} 4$ por cada muestreo. 
Debido a la dificultad de tomar la muestra tanto en la laguna de oxidación $\mathrm{N}^{\circ} 4$, como en el geotubo, se utilizó tubos PVC de 1 y 2 metros junto con corchos en las bocas superiores, de tal manera que se pueda extraer la muestra a presión y al vacío, a manera de una jeringa, absorbiendo el lodo, y manteniéndolo en el tubo. Esto sirvió para recoger la muestra de manera más eficiente a diferentes alturas.

Una vez tomadas las muestras fueron almacenadas en un cooler a una temperatura de $4^{\circ} \mathrm{C}$ y trasladadas a la ciudad de Quito. Una parte al laboratorio de la FIGEMPA (LABFIGEMPA) para realizar los análisis respectivos y la otra al laboratorio de Ciencias Químicas (OSP) para el análisis de la reactividad.

Las características de los lodos pueden variar en tiempos bastante prolongados como pueden ser en años, por ese motivo se realizó los muestreos y análisis a semana seguida. Por tanto, la investigación no se basó en cambios de características del lodo en el tiempo, sino más en la calidad del lodo bajo los parámetros ya explicados. Por este motivo la cantidad de muestras tomadas en los tres muestreos son suficientes para que sea representativo el análisis. Es decir, en la laguna de oxidación $\mathrm{N}^{\circ} 4$ se tomaron en total 9 submuestras, de las cuales se formaron 3 muestras compuestas y en el geotubo se tomaron 6 muestras en total.

\section{Diseño experimental}

Los parámetros analizados en las muestras recolectadas fueron los siguientes: humedad, nitrógeno total, fósforo total, potasio, pH para corrosividad, reactividad, toxicidad en base a metales pesados como plomo, níquel, cromo y cadmio, inflamabilidad, biológico infeccioso con sus respectivas metodologías que se detalla en la tabla 1.

Tabla 1. Parámetros y métodos utilizados en la caracterización de lodos

\begin{tabular}{|c|c|}
\hline \multicolumn{2}{|r|}{ Parámetros fisico - químico } \\
\hline Parámetro & Método \\
\hline Nitrógeno Total Kjeldahl & 8075 Nessler HATCH \\
\hline Fósforo & $\begin{array}{l}\text { EPA } 3050 \mathrm{~B} \text { método de análisis espectrofotométrico con } \\
\text { molibdato vanadato, método FIGEMPA }\end{array}$ \\
\hline Potasio & EPA 3050B; Standard Method 3500K-B \\
\hline Corrosividad $\mathrm{pH}$ & Standard Methods 4500- $\mathrm{H}^{+}, 9045 \mathrm{D}$ \\
\hline Reactividad & $\begin{array}{l}\text { Normativa Estándar del laboratorio OSP de Ingeniería } \\
\text { Química. }\end{array}$ \\
\hline $\begin{array}{l}\text { Metales pesados (niquel, } \\
\text { cromo plomo, cadmio) }\end{array}$ & $\begin{array}{l}\text { EPA 3050B, Standard Method 3111B y Standard Method } \\
\text { 3111D }\end{array}$ \\
\hline Inflamabilidad & Método estándar del laboratorio de Petróleos FIGEMPA \\
\hline Humedad & Método gravimétrico de incineración en mufla AOAC, 2005b \\
\hline \multicolumn{2}{|r|}{ Parámetros microbiológicos } \\
\hline Coliformes fecales & Método PETRIFILM AOAC, método oficial 991.14 \\
\hline Coliformes Totales & Método PETRIFILM AOAC, método oficial 991.14 \\
\hline
\end{tabular}

Fuente: Métodos citados

\section{Determinación de la humedad}

Para determinar el contenido de humedad se utilizó la metodología ACT-TE-INQM 13-12. Los equipos empleados fueron: balanza analítica, vasos de precipitación de $50 \mathrm{ml}$, paletas, estufa y desecador.

\section{Análisis NPK}

Determinación de nitrógeno total

La metodología empleada para la determinación del nitrógeno total se inició con el Método de Digestión para sólidos, procedimiento interno de laboratorio LABFIGEMPA, seguido de la preparación de la muestra con el método HACH 8075 para medición con el espectrofotómetro marca HACH, modelo DR/ 4000V.

\section{Determinación de fósforo total}

La metodología empleada para la determinación de fósforo total fue el Método de Digestión Ácida, procedimiento interno de laboratorio LABFIGEMPA, empleado para muestras ricas en materia orgánica y seguido de cálculo de factores de conversión a partir de la lectura de fosfato como P2O $5 \mathrm{mg} / \mathrm{L}$.

Los equipos e instrumentos empleados para esta metodología fueron: espectrofotómetro marca $\mathrm{HACH}$, modelo DR/ 4000V, balanza analítica marca RADWAG, balones de $50 \mathrm{ml}$, plancha eléctrica, embudo, papel filtro, vasos de $50 \mathrm{ml}$, tubos de ensayo, soporte universal y pipetas de 1, 5 y $10 \mathrm{ml}$.

\section{Determinación del potasio}

En la determinación del potasio se usó la metodología de Digestión Ácida procedimiento interno del laboratorio LABFIGEMPA, junto con la metodología de absorción atómica EPA 3050B como base y parte de la metodología Standard Method 3111B.

Los equipos y materiales que se usaron para este procedimiento fueron: espectrofotómetro de absorción atómica marca PerkinElmer, modelo PinAAcle 900T, balanza analítica marca RADWAG, balones de $50 \mathrm{ml}$, tapones para balones, soporte universal, embudos, papel filtro, pipetas de 1, 5 y $10 \mathrm{ml}$, vasos de $50 \mathrm{ml}$, plancha eléctrica y tubos de ensayo.

\section{Determinación de $\mathbf{p H}$ (corrosividad)}

Para la determinación de Potencial de Hidrógeno se empleó los Standard Methods 9045, método empleado para medir el $\mathrm{pH}$ en muestras de suelos o residuos sólidos, líquidos y lodos. El valor del pH nos indica el nivel de acidez o alcalinidad presente en una solución, demostrando la concentración alta o baja de iones de hidrógeno presentes.

Los equipos e instrumentos empleados para la determinación del $\mathrm{pH}$ fueron: potenciómetro marca InoLab 720 en perfectas condiciones y muy bien calibrada, balanza analítica marca RADWAG, agitadores y vasos de precipitación de $50 \mathrm{ml}$.

\section{Determinación de metales pesados (toxicidad)}

Para encontrar los metales pesados se usó los métodos de Digestión Ácida, procedimiento interno de laboratorio LABFIGEMPA, que se emplea para muestras que contengan gran cantidad de materia orgánica, seguido del método de absorción atómica APHA Method 3111B como guía para medición de metales.

Los equipos que se utilizaron en este procedimiento fueron: espectrofotómetro de absorción atómica marca PerkinElmer, modelo PinAAcle 900T, balanza analítica marca RADWAG, balones de $50 \mathrm{ml}$, pipetas 
de 1,5 y $10 \mathrm{ml}$, soporte universal, embudo, papel filtro, vasos de $50 \mathrm{ml}$, plancha eléctrica y tubos de ensayo.

El procedimiento es similar a la determinación de fósforo total en cuanto a la digestión, preparación de la muestra. Una vez terminado el proceso de la muestra se midió los metales pesados utilizando el espectrofotómetro de absorción atómica (Tabla 2).

Tabla 2. Lista de metales analizados en LABFIGEMPA

\begin{tabular}{ccccc}
\hline Parámetro & Unidad & Método & Equipo principal & $\begin{array}{c}\text { Límite de } \\
\text { detección }\end{array}$ \\
\hline Niquel & & & & 0,14 \\
Cromo & & & Espectrofotómetro de & 0,08 \\
Plomo & mgl & Ref. EPA 3050B & absorción atómica & 0,19 \\
Cadmio & & & PerkinElmer & 0,03 \\
Potasio & & & 0,04 \\
\hline
\end{tabular}

Fuente: LABFIGEMPA

Determinación de inflamabilidad

La medición de la inflamabilidad se basó con el método para determinar los puntos de inflamación y combustión mediante copa abierta de Pensky Martens (HFP 380 Flash Point Tester), del laboratorio de Petróleos en la facultad FIGEMPA, método guía utilizado para determinar los puntos de inflamación y combustión por medio de una copa abierta, para productos de petróleos, líquidos y en este caso muestras de suelos (ASTM Internaciona HACH, 2013). Es importante que el proceso se realice en un lugar libre de corrientes de aire con la finalidad que no altere su procedimiento.

Los equipos y materiales utilizados en esta práctica fueron: equipo de determinación de punto de inflamación Flash Point Tester marca HFP 380 Pnesky Martens, espátulas, termómetro, vasos de precipitación de $50 \mathrm{ml}$, tanque de gas industrial y fósforos.

\section{Análisis microbiológico}

En el parámetro microbiológico se usó la Guía 3M placas petrifilm, método oficial 991.14 para determinar a través del conteo las coliformes fecales y totales.

Los equipos utilizados en esta metodología fueron: pipeta automática, placas petrifilm, centrifugadora, incubadora y vasos de precipitación de $100 \mathrm{ml}$.

Debido a que la muestra es sólida, se necesitó la parte líquida de la mezcla para este análisis, sin que interfieran sólidos suspendidos en la muestra por lo cual se requirió centrifugar la muestra, colocando 25 $\mathrm{ml}$ de la mezcla en los tubos para centrifugado en la centrifugadora y ponerlo por 5 minutos a velocidad media.

De la muestra centrifugada se colocó $1 \mathrm{ml}$ con el uso de pipeta automática en las placas petrifilm de manera cuidadosa.

Las placas fueron etiquetadas con el número de muestra y colocadas en la incubadora con una temperatura de $30^{\circ} \mathrm{C}$ por unas 48 horas para proceder con el conteo e identificación de Coliformes totales.

\section{RESULTADOS}

Tabla 3. Registro de la humedad

\begin{tabular}{ccc}
\hline \multicolumn{3}{c}{ Muestreo del 28/08/2018 } \\
\hline Muestra & \%Humedad & Media \pm SD \\
\hline 1 & 87,03 & \\
2 & 88,89 & $87,47 \pm 1,262$ \\
3 & 86,49 & $87,74 \pm 1,721$ \\
\hline \multicolumn{3}{c}{ Muestreo del 04/09/2018 } \\
\hline 1 & 86,04 \\
2 & 87,69 & \\
3 & 89,49 & $89,62 \pm 0,366$ \\
\hline \multicolumn{3}{c}{ Muestreo del 11/09/2018 } \\
\hline
\end{tabular}

Parámetros NPK

Tabla 4. Registro del nitrógeno total kjeldahl

\begin{tabular}{ccccccc}
\hline Muestra & $\begin{array}{c}\text { Lectura } \\
(\mathbf{m g} / \mathbf{L})\end{array}$ & $\begin{array}{c}\text { P muestra } \\
(\mathbf{g})\end{array}$ & $\begin{array}{c}\text { Muestra seca } \\
(\mathbf{g})\end{array}$ & $\begin{array}{c}\text { Valor } \\
\mathbf{N T K} \\
(\mathbf{m g} / \mathbf{k g})\end{array}$ & $\begin{array}{c}\text { \% NTK } \\
(\mathbf{m g} / \mathbf{k g})\end{array}$ & $\begin{array}{c}\text { Niveles } \\
\text { críticos } \\
\mathbf{2 , 5 - 3} \%\end{array}$ \\
\hline 1 & 97,3 & 0,5626 & 0,073 & 50010,73 & 5,0 & Abundante \\
2 & 85,2 & 0,5112 & 0,057 & 56276,35 & 5,63 & Abundante \\
3 & 78,6 & 0,5587 & 0,075 & 39041,54 & 3,90 & Abundante \\
\hline \multicolumn{7}{c}{ Muestreo del 04/09/2018 } \\
\hline 1 & 103,6 & 0,5084 & 0,071 & 54751,15 & 5,47 & Abundante \\
2 & 77,5 & 0,5147 & 0,063 & 45880,43 & 4,58 & Abundante \\
3 & 71,3 & 0,5962 & 0,063 & 42651,82 & 4,26 & Abundante \\
\hline \multicolumn{7}{c}{ Muestreo del 11/09/2018 } \\
\hline 1 & 73,7 & 0,5525 & 0,060 & 46302,95 & 4,63 & Abundante \\
2 & 73 & 0,5298 & 0,054 & 50594,34 & 5,05 & Abundante \\
3 & 69,5 & 0,54 & 0,055 & 47640,80 & 4,76 & Abundante \\
\hline \multicolumn{7}{c}{ P muestra = peso de la muestra, NTK = nitrógeno total Kjeldahl }
\end{tabular}

Tabla 5. Registro de fósforo total

\begin{tabular}{|c|c|c|c|c|c|c|c|}
\hline \multicolumn{8}{|c|}{ Muestreo del 28/08/2018 } \\
\hline Muestra & $\begin{array}{c}\text { Lectura } \\
(\mathrm{mg} / \mathrm{L})\end{array}$ & $\underset{(g)}{P \text { muestra }}$ & $\begin{array}{c}\text { Ms en } \\
2(g) \text { de } \\
\text { muestra }\end{array}$ & $\begin{array}{c}(\mathrm{mg} / \mathrm{kg}) \\
\mathrm{P}_{2} \mathrm{O}_{5}\end{array}$ & $\begin{array}{c}(\mathrm{mg} / \mathrm{kg}) \\
\mathrm{P}_{\text {total }}\end{array}$ & $\begin{array}{l}\% \mathrm{P} \\
\text { total }\end{array}$ & $\begin{array}{c}\text { Niveles } \\
\text { criticos } \\
0,15-0,23 \\
\%\end{array}$ \\
\hline 1 & 20,4 & 2,00 & 0,26 & 98318,93 & 42927,98 & 4,29 & Abundante \\
\hline 2 & 22,96 & 2,09 & 0,23 & 123882,49 & 54089,53 & 5,41 & Abundante \\
\hline 3 & 20,52 & 2,33 & 0,32 & 81326,99 & 35508,97 & 3,55 & Abundante \\
\hline \multicolumn{8}{|c|}{ Muestreo del 04/09/2018 } \\
\hline 1 & 20,20 & 2,20 & 0,31 & 82121,11 & 35855,70 & 3,59 & Abundante \\
\hline 2 & 20,40 & 2,04 & 0,25 & 101568,21 & 44346,68 & 4,43 & Abundante \\
\hline 3 & 16,40 & 2,24 & 0,24 & 87113,31 & 38035,39 & 3,80 & Abundante \\
\hline \multicolumn{8}{|c|}{ Muestreo del 11/09/2018 } \\
\hline 1 & 17,70 & 2,06 & 0,22 & 99516,14 & 43450,71 & 4,35 & Abundante \\
\hline 2 & 15,40 & 2,02 & 0,21 & 93263,37 & 40720,62 & 4,07 & Abundante \\
\hline 3 & 16,50 & 2,27 & 0,23 & 89802,85 & 39209,70 & 3,92 & Abundante \\
\hline
\end{tabular}

Tabla 6. Registro del potasio

\begin{tabular}{ccccc}
\hline Muestreo & Muestra & $\mathbf{m g} / \mathbf{k g}$ & Porcentaje & Niveles críticos l-1,8\% \\
\hline \multirow{2}{*}{$28 / 08 / 2018$} & 1 & 12453,73 & 1,25 & Optimo \\
& 2 & 12388,25 & 1,18 & Optimo \\
& 3 & 12714,28 & 1,64 & Optimo \\
\hline \multirow{3}{*}{$04 / 09 / 2018$} & 1 & 11838,45 & 1,24 & Optimo \\
& 2 & 15135,66 & 1,51 & Optimo \\
& 3 & 16742,75 & 1,64 & Optimo \\
\hline \multirow{2}{*}{$11 / 09 / 2018$} & 1 & 16372,37 & 1,27 & Optimo \\
& 2 & 16375,59 & 1,67 & Optimo \\
& 3 & 21204,36 & 2,12 & Abundante \\
\hline
\end{tabular}




\section{Parámetros CRETIB}

Tabla 7. Registro de potencial de Hidrógeno (Corrosividad)

\begin{tabular}{|c|c|c|c|c|c|c|}
\hline \multicolumn{7}{|c|}{ Muestreo del 28/08/2018 } \\
\hline \multirow[b]{2}{*}{ Muestra } & \multirow[b]{2}{*}{ pH } & \multirow[b]{2}{*}{ Media } & \multicolumn{2}{|c|}{ Rango para corrosivo } & \multirow{2}{*}{$\begin{array}{c}\text { Temperatura } \\
\left({ }^{\circ} \mathrm{C}\right)\end{array}$} & \multirow[b]{2}{*}{ Media } \\
\hline & & & $\underset{\leq 2 . n \geq 12}{\text { N.n. }}$ & $\underset{\leq 20 \geq 12}{N . \text { in. }}$ & & \\
\hline 1 & 7,54 & \multirow{3}{*}{7,52} & No corrosivo & No corrosivo & 19,4 & \multirow{3}{*}{19,36} \\
\hline 2 & 7,55 & & No corrosivo & No corrosivo & 19,2 & \\
\hline 3 & 7,48 & & No corrosivo & No corrosivo & 19,5 & \\
\hline \multicolumn{7}{|c|}{ Muestreo del 04/09/2018 } \\
\hline 1 & 7,68 & \multirow{3}{*}{7,60} & No corrosivo & No corrosivo & 18,2 & \multirow{3}{*}{18,20} \\
\hline 2 & 7,6 & & No corrosivo & No corrosivo & 18,2 & \\
\hline 3 & 7,52 & & No corrosivo & No corrosivo & 18,2 & \\
\hline \multicolumn{7}{|c|}{ Muestreo del 11/09/2018 } \\
\hline 1 & 7,32 & \multirow{3}{*}{7,42} & No corrosivo & No corrosivo & 18,3 & \multirow{3}{*}{18,0} \\
\hline 2 & 7,43 & & No corrosivo & No corrosivo & 18,3 & \\
\hline 3 & 7.5 & & No corrosivo & No corrosivo & 17,4 & \\
\hline
\end{tabular}

Tabla 8. Registro de Cianuros (Reactividad)

\begin{tabular}{|c|c|c|c|c|c|c|}
\hline \multirow[b]{2}{*}{ Muestra } & \multirow[b]{2}{*}{$\begin{array}{c}\text { Masa de } \\
\text { muestra } \\
\left(K_{g}\right)\end{array}$} & \multirow[b]{2}{*}{$\begin{array}{c}\text { Concentración } \\
(\mathrm{mg} / \mathrm{Kg})\end{array}$} & \multirow[b]{2}{*}{ Media } & \multicolumn{3}{|c|}{ Limites permisibles } \\
\hline & & & & $\begin{array}{c}\text { N.n. } \\
<250 \mathrm{mg} \\
\mathrm{CN} / \mathrm{kg}\end{array}$ & $\begin{array}{c}\text { N. in. } \\
<250 \mathrm{mg} \text { CN } / \mathrm{kg}\end{array}$ & $\begin{array}{c}\text { N.n. uso } \\
\text { Agricola } \\
<0,9 \text { mg CN/kg }\end{array}$ \\
\hline 1 & 1 & 0,19 & & Cumple & Cumple & Cumple \\
\hline 2 & 1 & 0,09 & 0,1 & Cumple & Cumple & Cumple \\
\hline 3 & 1 & 0,02 & & Cumple & Cumple & Cumple \\
\hline
\end{tabular}

Tabla 9. Registro toxicidad por metales pesados

\begin{tabular}{|c|c|c|c|c|c|c|c|c|c|c|}
\hline \multirow[b]{2}{*}{ Muestreo } & \multirow[b]{2}{*}{ Parámetros } & \multirow[b]{2}{*}{$\begin{array}{c}\mathrm{M} \mathbf{1} \\
\mathrm{mg} / \mathrm{kg}\end{array}$} & \multirow[b]{2}{*}{$\begin{array}{c}\mathrm{M} 2 \\
\mathrm{mg} / \mathrm{kg}\end{array}$} & \multirow[b]{2}{*}{$\begin{array}{c}\mathrm{M} 3 \\
\mathrm{mg} / \mathrm{kg}\end{array}$} & \multicolumn{6}{|c|}{ Limites permisibles } \\
\hline & & & & & $\begin{array}{c}\text { N.n. } \\
\text { mg/kg }\end{array}$ & $\mathrm{C} / \mathrm{NC}$ & $\underset{\mathrm{mg}}{\mathrm{N} . \mathrm{in} .}$ & $\mathrm{C} / \mathrm{NC}$ & $\begin{array}{c}\text { A.M } \\
097 \\
\mathrm{mg} / \mathrm{kg}\end{array}$ & $\mathrm{C} / \mathrm{NC}$ \\
\hline \multirow{4}{*}{$28 / 08 / 2018$} & Niquel & 15,42 & 17,27 & 14,27 & 420 & $\mathrm{C}$ & 420 & $\mathrm{C}$ & 50 & $\mathrm{C}$ \\
\hline & Cromo & 50,12 & 56,11 & 52,32 & 1200 & C & 1200 & C & 65 & C \\
\hline & Plomo & - & - & - & 300 & C & 300 & $\mathrm{C}$ & 100 & $\mathrm{C}$ \\
\hline & Cadmio & - & - & - & 39 & $\mathrm{C}$ & 39 & C & 2 & C \\
\hline \multirow{4}{*}{$04 / 09 / 2018$} & Niquel & 21,14 & 21,91 & 29,75 & 420 & C & 420 & C & 50 & C \\
\hline & Cromo & 63,42 & 59,75 & 50,99 & 1200 & $\mathrm{C}$ & 1200 & $\mathrm{C}$ & 65 & C \\
\hline & Plomo & . & . & . & 300 & C & 300 & C & 100 & C \\
\hline & Cadmio & - & - & - & 39 & C & 39 & C & 2 & $\mathrm{C}$ \\
\hline \multirow{4}{*}{$11 / 09 / 2018$} & Niquel & 17,99 & 14,53 & 13,06 & 420 & $\mathrm{C}$ & 420 & $\mathrm{C}$ & 50 & $\mathrm{C}$ \\
\hline & Cromo & 60,72 & 50,87 & 43,54 & 1200 & C & 1200 & C & 65 & C \\
\hline & Plomo & 4,5 & 2,42 & 2,18 & 300 & C & 300 & C & 100 & C \\
\hline & Cadmio & - & - & - & 39 & C & 39 & C & 2 & C \\
\hline
\end{tabular}

Tabla 10. Registro de inflamabilidad

\begin{tabular}{ccccc}
\hline Muestra & $\begin{array}{c}\text { Masa de } \\
\text { muestra } \\
\text { seca (g) }\end{array}$ & $\begin{array}{c}\text { Punto de } \\
\text { inflamación }\end{array}$ & $\begin{array}{c}\text { Temperatura } \\
{ }^{\circ} \mathbf{C}\end{array}$ & $\begin{array}{c}\text { Gases inflamables e } \\
\text { inductores de explosión }\end{array}$ \\
\hline 1 & 50 & - & 90 & Sin presencia \\
2 & 50 & - & 90 & Sin presencia \\
3 & 50 & - & 90 & Sin presencia \\
\hline
\end{tabular}

Tabla 11. Registro de análisis microbiológico infeccioso

\begin{tabular}{|c|c|c|c|c|c|}
\hline \multicolumn{6}{|c|}{ Muestreo del 28/08/2018 } \\
\hline \multirow[b]{2}{*}{ Muestra } & \multirow{2}{*}{$\begin{array}{l}\text { No. } \\
\text { Coliformes } \\
\text { fecales }\end{array}$} & \multirow{2}{*}{$\begin{array}{l}\text { No. } \\
\text { Coliformes } \\
\text { Totales }\end{array}$} & \multirow{2}{*}{$\begin{array}{c}\text { No } \\
\text { Coliformes } \\
\text { totales final } \\
\text { (UFC/kg) }\end{array}$} & \multicolumn{2}{|c|}{ Límites permisibles } \\
\hline & & & & $\begin{array}{c}\text { N.n. } \\
2 * 10^{9} \mathrm{UFC} / \mathrm{kg}\end{array}$ & $\begin{array}{c}\text { N. in. } \\
2^{\star} 10^{9} \mathrm{UFC} / \mathrm{kg}\end{array}$ \\
\hline 1 & 2 & 2 & 3999,12 & Cumple & Cumple \\
\hline 2 & 2 & 3 & 5987,90 & Cumple & Cumple \\
\hline 3 & - & - & - & Cumple & Cumple \\
\hline \multicolumn{6}{|c|}{ Muestreo del 04/09/2018 } \\
\hline 1 & - & 2 & 3972,67 & Cumple & Cumple \\
\hline 2 & 3 & 10 & 19950,92 & Cumple & Cumple \\
\hline 3 & 3 & 14 & 27971,47 & Cumple & Cumple \\
\hline \multicolumn{6}{|c|}{ Muestreo del 11/09/2018 } \\
\hline 1 & - & 28 & 55843,64 & Cumple & Cumple \\
\hline 2 & 3 & 25 & 49109,16 & Cumple & Cumple \\
\hline 3 & - & - & - & Cumple & Cumple \\
\hline
\end{tabular}

\section{DISCUSIÓN}

\section{Caracterización del lodo}

La humedad de los lodos en el geotubo está en el rango de 86,04\% - 89,79\%, mientras que la humedad de los lodos en la laguna de oxidación $N^{\circ} 4$ es mayor y está entre $86,49 \%$ y $89,87 \%$. La función de deshidratar los lodos del geotubo no es eficiente en la actualidad, existe una mínima diferencia de humedad con la laguna de oxidación. Asimismo, el proceso de deshidratación lento, pues existen descargas de nuevos lodos por bombeo al geotubo y porque la membrana del geotubo no está limpia provocando que los microporos no permitan liberar el agua. Comparando con lo establecido en TENCATE GEOTUBE (2017), empresa creadora del geotubo y encargada de dar soluciones con geosintéticos, manifiesta que el geotubo tiene la función de remover el volumen de los lodos hasta un 90\%, con una humedad final por lo menos del $60 \%$, de este modo el volumen reduce, permitiendo que se vuelva a llenar nuevamente el geotubo con nuevos lodos. Además, en la revista TENCATE presenta casos de estudio donde se han aplicado el uso del geotubo, como por ejemplo; la "Limpieza de laguna de granja" en el sector de New Bern, Carolina del Norte, dando resultados de reducción de la humedad de los lodos hasta un $25 \%$ y removiendo hasta el $93 \%$ de los mismos. (TENCATE, 2007).

\section{Parámetros agrícolas $(\mathbf{N}, \mathbf{P}, \mathbf{K})$}

La relación de nitrógeno total de los lodos de la laguna de oxidación está en el rango de 3,90\% y 4,76 , mientras que en el geotubo el rango de nitrógeno total está entre 4,58\% y 5,63\%, no existe una diferencia considerable entre los lodos de la laguna de oxidación y el geotubo. Estos resultados sobrepasan los límites máximos de nitrógeno en comparación con estudios previos de investigación realizadas por (Owen, 2011), ya que, para emplear los lodos en la fertilización del suelo no debe exceder el rango de $0,1 \%$ a 0,4\%.

Según estudios realizados, la alta concentración de nitrógeno se debe a que, en regiones de clima tropical, la alta temperatura hace que el desarrollo de reacciones, principalmente de nitrificación, sea inevitable. Es decir, por la descomposición de la alta concentración de materia orgánica se forman nitratos, amoniaco y amonio. Además, debido al proceso anaerobio no se lleva a cabo la remoción de nitrógeno, una prueba de esto es la eutrofización que se visualiza en la laguna.

La relación de fósforo en la laguna de oxidación está entre 3,55\% - 3,92\%, mientras que en el geotubo el fósforo tiene una concentración entre 4,07\% $5,41 \%$. Los resultados de fósforo comparados con valores de diferentes autores citados por (Owen, 2011) son elevados, debido a que en su guía de fertilización de palma africana los valores críticos de fósforo en suelo están entre 0,15\% y 0,23\%.

La alta concentración de fósforo en los lodos se debe a la acumulación y descomposición de 
la materia orgánica, provocando el exceso de nutrientes en la laguna de oxidación, entre estos el fósforo. La evidencia de esto es la eutrofización que se visualiza en la laguna de oxidación y como no se ha encontrado ningún cambio en su composición, el momento del traspaso del lodo hacia el geotubo, tendrá la misma tendencia de concentración de fósforo que en la laguna.

Las concentraciones de potasio en los lodos para la laguna de oxidación están entre 1,64\% y 2,12\%, en cambio, en el geotubo oscila entre 1,18\% y 1,67\%, con una diferencia mínima tanto para la laguna como para el geotubo. De los datos encontrados según investigaciones citadas por (Owen, 2011), los porcentajes adecuados para una fertilización deben estar entre $1 \%$ y $1,8 \%$ de potasio.

El potasio es el elemento que más absorbe la planta, por ende, se encuentra en todos los tejidos de la palma africana, en consecuencia, es factible deducir que los residuos que vienen de la extractora y van tanto a la laguna de oxidación como al geotubo tienen alta concentración de potasio.

La relación de NPK de los lodos es aproximadamente de 3:3:1, siendo así más factible usarlo como fertilizante cuando las plantaciones de palma tengan déficit de nitrógeno y fósforo.

\section{Potencial hidrógeno (corrosividad)}

En los tres muestreos realizados tanto en la laguna de oxidación como en el geotubo, el pH estuvo entre 7,43 y 7,68 indicando que los lodos tienen un $\mathrm{pH}$ ligeramente neutro. Estos valores van de acuerdo con las normativas ambientales nacionales (Resolución Nacional 0002-SA-2014) e internacionales (NOM052-SEMARNAT-2005) donde se indica que el rango para que este material sea corrosivo debe ser menor o igual a 2 y mayor o igual a 12, corroborado según los estudios realizados por Soler (2011) "el pH es relativamente neutro debido a que en el proceso de extracción no se utilizan productos químicos que modifiquen las propiedades físico químicas de los lodos, por tanto, no se altera el $\mathrm{pH}$ natural del agua" y en el tratamiento de las aguas no se introduce ningún tipo de agente químico, resultando un lodo con $\mathrm{pH}$ neutro.

\section{Análisis de reactividad de los lodos}

En el análisis de reactividad en base a la concentración de cianuros se obtuvo valores bastante despreciables, como, 0,02 mg CN/kg en la laguna de oxidación $\mathrm{N}^{\circ} 4$ y en el geotubo valores entre 0,09-0,19 mg CN/kg. Si comparamos estos resultados con los valores establecidos por la norma oficial mexicana NOM-052-SEMARNAT-2005, la concentración de cianuro no debe sobrepasar los $250 \mathrm{mg}$ CN/kg. Además, en el A.M. 097 de criterios para uso y remediación de suelo, se establece que el límite permisible para usos agrícolas debe ser de $0,9 \mathrm{mg} \mathrm{CN} / \mathrm{Kg}$. No existen investigaciones similares de este caso de estudio para reactividad en lodos, pero se tiene evidencias de reportes de dicho análisis realizados en los lodos del sistema de tratamiento de aguas residuales de la industria OLEOCASTILLO, confirmando que no hubo reacción visible de reactividad en sus lodos (GRUNTEC, 2012).

\section{Análisis tóxico por metales pesados}

La concentración de níquel encontrada en los lodos de la laguna de oxidación $N^{\circ} 4$, a partir de los tres días de muestreo, está en un rango de 13,06$29,75 \mathrm{mg} / \mathrm{kg}$ y la concentración en el lodo del geotubo está en el rango de 14,53-21,91 mg/kg, si comparamos con los límites máximos permisibles para metales pesados en biosólidos de la normativa NOM-004-SMART-2002, la concentración de 420 $\mathrm{mg} / \mathrm{kg}$ de níquel es catalogada como excelente, lo que indica que el lodo analizado tanto en la laguna de oxidación como en el geotubo tiene una concentración mínima en relación a la norma. También, según los criterios del A.M. 097, para uso y remediación de suelo, el níquel tiene que estar hasta un nivel de $50 \mathrm{mg} / \mathrm{kg}$ para uso agrícola y los lodos analizados se encuentran bajo el nivel mencionado.

Respecto al cromo, para la laguna de oxidación $\mathrm{N}^{\circ} 4$, se encuentra entre $43,54-52,32 \mathrm{mg} / \mathrm{kg}$ y en el geotubo entre $50,12-63,42 \mathrm{mg} / \mathrm{kg}$, no hay una diferencia tan apreciable entre los valores de la laguna y del geotubo. Asímismo, comparando los datos establecidos de la normativa NOM004-SMART-2002, nos da un valor de 1200 mg/ $\mathrm{kg}$ de cromo catalogado como un nivel excelente para lodos, con los resultados de cromo analizado podemos decir que tiene una concentración mínima. De la misma manera, comparando con los valores del A.M. 097 en criterios para uso y remediación de suelo, el cromo puede ser catalogado para uso agrícola ya que su concentración no sobrepasa los $65 \mathrm{mg} / \mathrm{kg}$ recomendados.

En los dos primeros muestreos del lodo realizados no se detectó presencia de plomo tanto en la laguna de oxidación $\mathrm{N}^{\circ} 4$ como en el geotubo. Sin embargo, el tercer muestreo presentó un valor de 2,18 mg/kg para la laguna de oxidación y valores entre 2,42-4,50 $\mathrm{mg} / \mathrm{kg}$ para el geotubo. De acuerdo con la normativa NOM-004-SMART-2002 el plomo debe estar bajo los límites de 300 mg/kg y según el A.M. 097 para uso agrícola y para remediación de suelo el plomo debe estar en un nivel de $100 \mathrm{mg} / \mathrm{kg}$.

Una de las causas por las que no se detectó plomo en los dos primeros muestreos fue debido a las interferencias que se pueden presentar, ya que en el análisis del tercer muestreo la cantidad de plomo es bastante despreciable y por la heterogeneidad de la muestra y la dificultad de homogeneizar el lodo en la laguna de oxidación y en el geotubo.

No se evidenció la presencia de cadmio en los tres muestreos realizados tanto en la laguna de oxidación $\mathrm{N}^{\circ} 4$ y en el geotubo. Las concentraciones son mínimas en el lodo, ya que el límite de detección del espectrofotómetro es hasta 0,03mg/l.

\section{Análisis de inflamabilidad de los lodos}

En este análisis, método cualitativo para los lodos de la laguna de oxidación $\mathrm{N}^{\circ} 4$ y del geotubo, no presentó 
ningún indicio de reacción inflamable, confirmando que no existen vapores inflamables capaces de causar un incendio en el lodo. Según estudios realizados por (Esparza, 2019) "ningún cuerpo arde en su masa o volumen, sino que lo que se quema son los vapores que se desprenden al alcanzar las temperaturas de inflamación", en este caso solo se desprendió vapores de agua por la humedad que presenta el suelo siendo estos inertes a causar alguna reacción inflamable corroborando los resultados obtenidos de la investigación. Por otro lado, tomando en cuenta estudios del parámetro de inflamabilidad que se han realizado con anterioridad en lodos del sistema de tratamiento de aguas residuales de la industria OLEOCASTILLO, no se han presentado vapores inflamables de los mismos. (GRUNTEC, 2012).

\section{Análisis microbiológico de los lodos}

Es difícil hacer el análisis microbiológico de lodos ya que se necesita una sustancia libre de material suspendido para realizar la siembra de microorganismos, este material suspendido causa interferencia en la visualización de los microorganismos plantados. Sin embargo, se realizó el análisis evitando al máximo estas interferencias, pero en sí, son valores que están por debajo de los límites permitidos que dicta la Resolución nacional No. 0002-SA-2014, 2014 y la normativa internacional NOM-004-SEMART-2002, 2002. Ahora, tomando en consideración investigaciones sobre análisis microbiológicos, los valores obtenidos son similares con el estudio realizado por Malacatus, Paredes y Chisaguano (2017) en "Análisis de generación características tóxicas y biológico infecciosas de lodos de la Potabilizadora los Álamos, observando que sus resultados obtenidos de criterios microbiológicos se encuentran bajo los límites permisibles", mientras que en análisis de laboratorio realizado por GRUNTEC (2012) en el sistema de tratamiento de aguas industriales de la industria OLEOCASTILLO, se apreció resultados de parámetros microbiológicos bajo los límites permisibles.

\section{CONCLUSIONES}

Con respecto al nitrógeno y fósforo total del lodo tanto en la laguna de oxidación como en el geotubo, sobrepasan los límites críticos óptimos de la guía de fertilización que se cita: para nitrógeno 2,5 \% - 3\% y para fósforo de 0,15\% - 0,23\%. Por otro lado, las concentraciones de potasio se encuentran entre los niveles críticos de $1 \%-1,8 \%$, es decir tiene una concentración óptima. Por este motivo se determinó que el lodo es rico en nutrientes, ideal para utilizarlo como fertilizante de la plantación de palma; los datos obtenidos en el laboratorio nos dan una relación N, P, K de 3:3:1 para el lodo.

El lodo tanto en la laguna de oxidación $\mathrm{N}^{\circ} 4$ como en el geotubo tienen un $\mathrm{pH}$ que varía de 7,43 - 7,68 lo que significa que el lodo no es corrosivo, pues se encuentra fuera del rango de corrosividad.

Para la reactividad, la concentración de cianuro en el lodo de la laguna de oxidación $\mathrm{N}^{\circ} 4$ es de 0,02 $\mathrm{mg} \mathrm{CN} / \mathrm{kg}$ y en el geotubo es de 0,09-0,19 mg CN/ $\mathrm{kg}$, por tanto, no son un material reactivo, ya que es menor a $250 \mathrm{mg} \mathrm{CN} / \mathrm{Kg}$, mucho menos del límite permisible. De igual manera, puede ser empleado para uso agrícola según el A.M. 097 debido a que no supera los límites de 0,9 mg CN/Kg.

En la toxicidad por metales pesados de los lodos tanto en la laguna de oxidación $\mathrm{N}^{\circ} 4$ como en el geotubo, los resultados de los metales níquel, cromo, plomo y cadmio, están bajo los límites permisibles según las normativas vigentes; es decir, el lodo no es toxico.

En la inflamabilidad de los lodos de la laguna de oxidación $\mathrm{N}^{\circ} 4$ y del geotubo, no se registra la presencia de gases volátiles inflamables ni inductores de explosión, por lo tanto se determinó que no es inflamable.

Los análisis microbiológicos en base a unidades formadoras de coliformes totales, indican valores por debajo de los límites establecidos por las normativas nacionales e internacionales, con lo cual se concluye que los lodos analizados son catalogados como desechos biológicos no peligrosos.

Por los resultados de los análisis CRETIB obtenidos, comparando con la Legislación Ambiental vigente nacional e internacional se comprueba que el lodo no es peligroso.

Como no existe una diferencia significativa entre los valores de la caracterización de los lodos de la laguna de oxidación y del geotubo, se puede establecer que el polímero no causa ningún tipo de cambios en sus componentes fisicoquímicos, biológicos y agrícolas.

Los lodos son considerados no peligrosos por lo que su destino final puede ser un relleno sanitario sin causar afectación ambiental.

\section{RECOMENDACIONES}

Realizar un análisis de metales pesados cada determinado tiempo, ya que, la concentración de cromo se encuentra muy cerca del límite permisible según el A.M. 097 y no puede ser recomendable directamente para uso agrícola.

Emplear un procedimiento para disminuir la concentración de metales pesados si la situación lo requiere.

Realizar un análisis del procedimiento para una buena dosificación del lodo en cantidades necesarias hacia utilizarlo como fertilizante. El procedimiento debe contar con un tratamiento para los lodos que dependerá mucho de las características requeridas por la plantación de palma a fin de que cumplan con los requisitos y parámetros necesarios.

Se puede mezclar este lodo con otros materiales para disminuir las concentraciones de nitrógeno y fósforo, si se requiere, después del análisis que se realice para distribuir el lodo y según las necesidades de la plantación.

Una vez que los lodos son considerados como no peligrosos, se puede también trasladarlos a rellenos 
sanitarios autorizados, pues el impacto ambiental será mínimo y puede formar parte del relleno sanitario.

Mantener la limpieza de la membrana del geotubo, tratando de que no crezca maleza en las paredes del geotubo para que sea más eficiente la deshidratación de los lodos.

\section{REFERENCIAS}

- ASTM Internaciona HACH, (2013). Manual de análisis de agua.

- Acuerdo Ministerial 097 (2015). "Anexo 2 del libro VI del texto unificado de legislación secundaria del misterio del ambiente: Normas de calidad ambiental del recurso suelo y criterios de remediación para suelos contaminados" en Ministerio del Ambiente. [En línea]. Ecuador, disponible en: http://gis.uazuay.edu.ec/ierse/ links_doc contaminantes/REGISTRO\%20 OFICIAL\%20387\%20-\%20AM\%20140.pdf. Revisado: 10/10/2018.

- Chisaguano, W., Malacatus, P., y Paredes, Y. (2017). Análisis de generación características tóxicas y biológico infecciosas de lodos de la potabilizadora los Álamos. FIGEMPA Investigación y desarrollo, 7.

- Cogollo, J. (2010). Clarificación de aguas usando coagulantes polimerizados: caso del hidroxicloruro de aluminio. Revista DYNA, vol. 78, No. 165, 20.

- Esparza, F., (s.f.) "Combustibles Sólidos, Líquidos y Gaseosos" en BS. España, Bomberos de Navarra Nafarroako Suhiltzaileak.

- GRUNtek Environmental Services, (2012), Reporte de análisis. Ecuador, GRUNtek Environmental Services.

- HACH., (2000). "Manual de análisis de agua" en HACH COMPANY. [En línea]. EE. UU., Disponible en: https://www.hach.com/asset-get.download. jsa?id=7639984469. Revisado: 19/09/2018.

- Instituto Mexicano de tecnología del agua. (2000). Tratabilidad de los lodos producidos en la potabilización del agua en coordinación de tratamiento y calidad de Agua. México.

- Malacatus, P., Paredes, Y., y Chisaguano, W., (2017). "Análisis de generación - características tóxicas y biológico infecciosas de lodos de la Potabilizadora los Álamos" en FIGEMPA: INVESTIGACIÓN Y DESARROLLO. Año II, vol. I, No. 1. Enero-marzo 2017, pP. 5-6.

- NOM-004-SEMARNAT-2002, (s.f.)."Protección ambiental. - Lodos y biosólidos. - Especificaciones y límites máximos permisibles de contaminantes para su aprovechamiento y disposición final. México.

- Norma Oficial Mexicana. NOM-052-
SEMARNAT-2005. "Características, procedimiento de identificación, clasificación y los listados de residuos peligrosos" en Diario oficial de la Federación. [En línea]. México, disponible en: http://www.inb.unam.mx/stecnica/nom052_ semarnat.pdf. Revisado: 05/07/2018.

- Norma Oficial Mexicana. NOM-004SEMARNAT-2002. "Protección ambiental. Lodos y biosólidos. - Especificaciones y límites máximos permisibles de contaminantes para su aprovechamiento y disposición final" en Diario oficial de la Federación. [En línea]. México, disponible en: http://legismex.mty.itesm.mx/ normas/ecol/semarnat004.pdf. Revisado: 18/07/2018.

- Owen, E. (2011). Fertilización de la palma africana (ElaeisGuineensisJacq.) en Colombia. Revista Palmas. Volumen 13, número 21992., 40-49.

- Pérez, M. (2016). Tratamiento de lodos residuales procedentes de planatas de tratamiento de aguas residuales mediante procesos electroquímicos para la disminución de metales. Tesis de ingeniería. Ecuador, Carrera de ingeniería ambiental, Universidad Politécnica Salesiana., 150.

- Secretaría de Ambiente del Municipio de Quito, (2014). Resolución Nacional 0002-SA-2014. Quito.

- Soler, F., (2018). "Aceite de Palma: Cultivo, Procesos de Obtención, Refinación y Transformación" en LIPSA [En línea]. Ecuador, disponible en: http://www.aiqs.es/uploads/managearticle/7/2018/03/16/20180316120421-0559. pdf

- TENCATE Gotube (2017) "La solución de deshidratación de alto volumen y bajo costo" en Soluciones de desagüe ambiental. [En línea]. Georgia, disponible en: https:// www.tencategeotube.com/en/solutions/ environmental-dewatering

- TENCATE Geotube (2007) "textiles industriales Sistemas de filtración" en Tecnología de filtración Geotube. [En línea]. Georgia, disponible en: https:// es.slideshare.net/slidesharedgp/geotubostencate-secado-de-lodos

- UNASAM - FCAM. (2009). Procedimiento de muestreo de agua superficial. Sistema de gestión de calidad - NTP ISO/IEC 17025. 